\title{
Traction Inverter Open Switch Fault Diagnosis Based on Choi-Williams Distribution Spectral Kurtosis and Wavelet-Packet Energy Shannon Entropy
}

\author{
Shuangshuang Lin ${ }^{1,2}$, Zhigang Liu ${ }^{1,2, *}$ (1) and Keting $\mathrm{Hu}^{1,2}$ \\ 1 Key Laboratory of Magnetic Suspension Technology and Maglev Vehicle, Ministry of Education, \\ Southwest Jiaotong University, Chengdu 610031, China; shuangshuang_lin@sina.cn (S.L.); \\ huketing@my.swjtu.edu.cn (K.H.) \\ 2 School of Electrical Engineering, Southwest Jiaotong University, Chengdu 610031, China \\ * Correspondence: liuzg_cd@126.com; Tel.: +86-28-8760-3229
}

Received: 4 September 2017; Accepted: 11 September 2017; Published: 16 September 2017

\begin{abstract}
In this paper, a new approach for fault detection and location of open switch faults in the closed-loop inverter fed vector controlled drives of Electric Multiple Units is proposed. Spectral kurtosis (SK) based on Choi-Williams distribution (CWD) as a statistical tool can effectively indicate the presence of transients and locations in the frequency domain. Wavelet-packet energy Shannon entropy (WPESE) is appropriate for the transient changes detection of complex non-linear and non-stationary signals. Based on the analyses of currents in normal and fault conditions, SK based on CWD and WPESE are combined with the DC component method. SK based on CWD and WPESE are used for the fault detection, and the DC component method is used for the fault localization. This approach can diagnose the specific locations of faulty Insulated Gate Bipolar Transistors (IGBTs) with high accuracy, and it requires no additional devices. Experiments on the RT-LAB platform are carried out and the experimental results verify the feasibility and effectiveness of the diagnosis method.
\end{abstract}

Keywords: open switch fault; Shannon entropy; spectral kurtosis (SK); traction inverter; fault diagnosis

\section{Introduction}

In recent years, the rapid development of high-speed railways has drawn attention to the security and stability requirements of operation. In the operation process of Electric Multiple Units, sensor failures, open-circuit and short-circuit faults of converter power devices could easily occur due to the complex and changeable environment, which could threat the operational safety of trains [1].

Presently, closed-loop inverters are applied widely in engineering projects, and they realize their function by semiconductor power devices (SPDs). An earlier survey has pointed out that SPDs are ranked among the most fragile components in power electronic converters [2]. SPD failures include open switch faults and short switch faults. For the short switch fault condition, the time between fault initiation and failure is short, thus, it is generally protected by hardware. For the open switch fault condition, they are not only difficult to find, but also cause additional failures and damage to the system [3]. Hence, it is of great importance to diagnose the open switch faults in a timely way.

Numerous methods have been proposed for the diagnosis of open switch faults, which can be classified into current-based and voltage-based methods. Generally, voltage-based methods realized the detection by comparisons of the faulty motor phase voltage or line voltage of the inverter with a normal reference voltage [4,5]. In addition, the diagnosis was performed by integrating the voltage with intelligent algorithms, such as artificial neural network [6]. However, voltage-based methods 
need additional voltage sensors, and are easily influenced by system noise, therefore, voltage-based open switch fault diagnostic algorithms are rarely used in inverters.

Wavelet, Concordia and Park transform methods are commonly adopted in current-based methods. In [7], wavelet and Concordia transforms were combined to acquire the current track as the characteristic, and diagnosis was implemented through a classical fault dictionary and support vector classifier methods. In [8,9], multi-resolution based on wavelet transforms was adopted and the features of open switch faults were discussed and applied to the diagnosis. However, it was discussed in [10] that a sudden change of current amplitude could influence the diagnosis result, which might cause false alarms and make it difficult to realize the diagnosis by wavelet transform. As for the Concordia and Park transform methods, analyses of the fault-free and fault conditions were shown in [11]. It adopted average absolute values of phase currents based on the Park vector to formulate the diagnostic variable. In [12], a new way for Insulated Gate Bipolar Transistor (IGBT) open switch faults diagnosis in pulse-width modulation voltage-source inverters was presented. It was based on the distortions of the current distribution in the $d-q$ frame combined with normalized average current values. In [13], an average current Park vector approach was proposed. It was compared with the set threshold to detect the faults and realize the diagnosis. Although this diagnostic method had strong robustness, it relied on loads and was hard to apply in actual systems. It was also argued in [14] that the Park transform method would increase the diagnosis time. The paper put forward the kernel density estimation instead of the Park transform method, and the results showed the effectiveness of proposed method.

Based on the above discussion, wavelet, Concordia and Park transform methods would not be the good ways to deal with open switch faults of IGBTs in traction inverters. As the signal processing (SP) methods have shown great potential in the engineering field $[15,16]$, an attempt to diagnose open switch faults of IGBTs by SP is made in this paper. Considering that the transient process contains abundant fault information, SP methods that can detect the transient processes could be very useful. For example, reference [17] utilized the dynamic Bayesian wavelet transform to reveal fault signatures hidden in rotating machine signals by detecting the repetitive transients. In [18], the enhanced kurtogram was utilized to realize spectra kurtosis (SK) faster and diagnose various bearing faults by extracting the transient information, despite the noise. In [19], an improved SK is proposed to locate the transients in the frequency domain under heavy noise conditions, and then the early faults of bearings can be detected. Due to the fact that SK shows good anti-noise performance, it is adopted in this paper. The concept of SK was first proposed by Dwyer, who used it for detection of transient components in noisy signals [20]. In [21], Antoni defined SK systematically and presented SK based on short time Fourier transform (STFT). It was demonstrated that SK based on STFT had the ability to detect transient signals in nonstationary signals with additive noise. In [22], SK based on STFT, wavelet transform (WT), Wigner-Ville distribution (WVD) and Choi-Williams distribution (CWD) were discussed and different parameters about SK based on CWD were analyzed. The paper put forward a new method compared with thresholds to realize the detection of disturbances in power quality.

Recently, many algorithms combined with entropy have shown good performance in the diagnosis of faults [23-28]. In [23], negentropy was utilized to extend SK, which was able to detect impulsive and cyclostationary transients of bearing faults. In [24], combined with the hidden Markov model, the multi-scale permutation entropy was proposed to identify the fault types of bearings. In [25], it pointed out that the healthy condition of the trajectories can be determined by a measure based on Shannon entropy. In [26], wavelet packet entropy was applied to the recognition of combined power quality disturbances. Multi-wavelet singular entropies were proposed to detect and classify faults in power transmission lines in [27]. In [28], five entropy forms were put forward and discussed. The values of entropy were calculated and combined with DC components to detect the specific locations of fault IGBT or IGBTs in different open switch fault conditions. 
Considering the good performance of SK and entropy in diagnosis of faults, a new approach for vector controlled induction motor drives is presented in this paper. This method consists of two parts: the fault detection and fault location. For the fault detection part, SK based on CWD is utilized to detect the faults and combined with wavelet-packet energy Shannon entropy (WPESE) method to determine the fault phase. Then for the fault location part, DC components of the fault phase are calculated and compared to identify the upper switch fault (USF) and the lower switch fault (LSF). This approach could solve the false alarm problem which would be caused by the system noise and has good robustness. Compared with some previous methods, it can realize the diagnosis of all single and two IGBTs fault cases, which is easily-operated and requires no additional devices. Finally, experiments on the RT-LAB platform are carried out, and the results verify the effectiveness of the proposed approach.

\section{Spectral Kurtosis Based on CWD and Wavelet-Packet Energy Shannon Entropy}

\subsection{Spectral Kurtosis Based on Choi-Williams Distribution}

As a statistical tool, SK can detect the non-Gaussian components in a signal, which can indicate the presence of transients and their locations in the frequency domain. Once the open switch fault of an IGBT occurs, the harmonic components in fault currents that are different from the main frequency components would increase, thus, the amplitude of SK in the frequency domain would get increased correspondingly. Therefore, SK is adopted in this paper to detect faults. There are many algorithms combined with SK, such as STFT, WT, WVD [22]. SK based on STFT shows a low time-frequency resolution, and it is limited to the choice of window functions. Besides, the results are poor when the system is influenced by much noise. SK based on WT has a strong ability to acquire the characteristics in the time and frequency domain, however, the wavelet bases and decomposition scales are difficult to determine, which cannot get the most excellent diagnosis results. SK based on WVD has many good properties, but the cross-term in the signals cannot be completely eliminated, and it affects the analyses of results. In order to solve the cross-term problem, SK based on CWD is developed. It inherits the excellent time-frequency performance of WVD, and suppresses the interference of the cross-term effectively. Therefore, this paper adopts SK based on CWD method to recognize the fault conditions.

For a nonstationary signal $x(t)$, based on CWD algorithm, $C_{x}(t, f)$ can be presented below [29]:

$$
C_{x}(t, f)=\iint \sqrt{\frac{\sigma}{4 \pi \tau^{2}}} \exp \left(-\frac{\sigma t^{2}}{4 \tau^{2}}\right) x\left(\mu+\frac{\tau}{2}\right) x^{*}\left(\mu-\frac{\tau}{2}\right) e^{-j 2 \pi f \tau} d \mu d \tau
$$

where, $\tau$ is the time shift parameter, $\sigma$ is the scale factor and $\mu$ represents the partial time.

In CWD algorithm, the exponential kernel function $g(\theta, \tau)=\exp \left(-\frac{\theta^{2} \tau^{2}}{\sigma}\right)$ is adopted. $g(0, \tau)=g(\theta, 0)=1, g(0,0)=1$, and when $\theta \neq 0, \tau \neq 0, g(\theta, \tau)<1$, where, $\theta$ represents the frequency offset parameter, and $\sigma$ is a constant. If $\sigma$ gets large, the resolution would become higher. If $\sigma$ gets small, the inhibitory effect of cross-term would become stronger. Thus, $\sigma$ should be determined by considering the two aspects. Therefore, the exponential kernel function can suppress the interference of the cross-term and show high time-frequency performance.

The second order and fourth order spectral moments also can be shown as follows:

$$
\left\{\begin{array}{l}
\hat{S}_{2 x}(f)=E\left\{\left|C_{x}(t, f)\right|^{2}\right\}_{k} \\
\hat{S}_{4 x}(f)=E\left\{\left|C_{x}(t, f)\right|^{4}\right\}_{k}
\end{array}\right.
$$

where, $E\{\bullet\}_{k}$ represents the average of $k$-th order.

Finally, based on the definition of SK [21], SK based on CWD is defined below:

$$
K_{x}(f)=\frac{C_{4 x}(f)}{\hat{S}_{2 x}^{2}(f)}=\frac{\hat{S}_{4 x}(f)-2 \hat{S}_{2 x}^{2}(f)}{\hat{S}_{2 x}^{2}(f)}=\frac{\hat{S}_{4 x}(f)}{\hat{S}_{2 x}^{2}(f)}-2,(f \neq 0)
$$




\subsection{Wavelet-Packet Energy Shannon Entropy}

The Shannon entropy was proposed to solve the problem of quantitative measurement of information by Shannon. Once a fault occurs in the inverter, more frequency components would appear in three-phase currents. Thus, the value of entropy in a fault case is more than that in a healthy condition, which is appropriate for diagnosis of open switch faults.

Compared with the wavelet transform, the wavelet-packet transform has a high resolution to decompose the detail part in each level, which is more fit for the detection of fault [28]. The recursive decomposition of discrete wavelet-packet can be expressed as follows:

$$
\begin{aligned}
& d_{i, 2 j}(k)=\sqrt{2} \sum_{k} g(k) d_{i-1, j}(2 t-k) \\
& d_{i, 2 j-1}(k)=\sqrt{2} \sum_{k} h(k) d_{i-1, j}(2 t-k) \\
& d_{0,0}(k)=f(x)(t)
\end{aligned}
$$

where, $k$ is the index of sampling point, $f(x)$ represents the original signal, $g(k)$ and $h(k)$ are the low pass filter and high pass filter respectively, and $d_{i, j}(k)$ is the reconstructed signal of wavelet packet decomposition for the $j$-th node at the $i$-th level.

Supposing a random variable $X\left(X=X_{1}, X_{2}, \ldots, X_{N}, N\right.$ is the number of probable states $)$ as the state of an uncertain system, the definition of Shannon entropy is shown below:

$$
H(X)=-\sum_{j=1}^{N} p_{j} \log \left(p_{j}\right)
$$

where, $p_{j}$ is the probability of $x_{i}$, and $\sum_{j=1}^{N} p_{j}=1$.

Based on the wavelet-packet transform, the wavelet-packet energy can be obtained by the Equation (6), and the total energy $E_{j}$ is the sum of wavelet-packet energy of every node. Hence, its probability can be acquired in Equation (7), and WPESE is defined as Equation (8):

$$
\begin{gathered}
E_{i, j}=\sum_{n=1}^{k}\left|d_{i, j}(k)\right| \\
p_{i, j}=\frac{E_{i, j}}{E_{j}} \\
\text { WPESE }=-\sum_{i=1}^{J} p_{i, j} \log p_{i, j}\left(J=2^{j}\right)
\end{gathered}
$$

\section{Diagnosis Plan}

\subsection{Analyses of Faults and Diagnosis Plan}

The schematic diagram of a traction system is shown in Figure 1. The vector controlled traction inverter mainly consists of six SPDs, namely $S 1, S 2, S 3, S 4, S 5$, S6. IGBT with antiparallel diode is a kind of commonly adopted SPD [30]. In this paper, compared with the open-loop system, the closed-loop system is adopted, and it has a feedback loop, which can be utilized to detect the control output and then revise control errors. As a consequence, the closed-loop system has an ability of restraining interference and improving the response of the system. However, if an IGBT fails, the output currents of other phases would be influenced, which would add much difficulty to the fault diagnosis.

There are many fault conditions of open switch faults with IGBTs in the closed-loop inverter, and the probability that three IGBTs have open switch faults simultaneously is very small. Consequently, 
this paper assumes that two IGBTs fail at the same time at the most. Thus, open switch failure types of IGBTs have the following four major categories:

(a) Fault A case: Only an IGBT has a fault, such as S1;

(b) Fault B case: Two IGBTs located on the different side of the half bridge arm have faults simultaneously, such as $S 1, S 6$;

(c) Fault $C$ case: Two IGBTs in the single phase have faults simultaneously, such as S1, S4;

(d) Fault D case: Two IGBTs located on the same side of the half bridge arm have faults simultaneously, such as $S 1, S 3$.

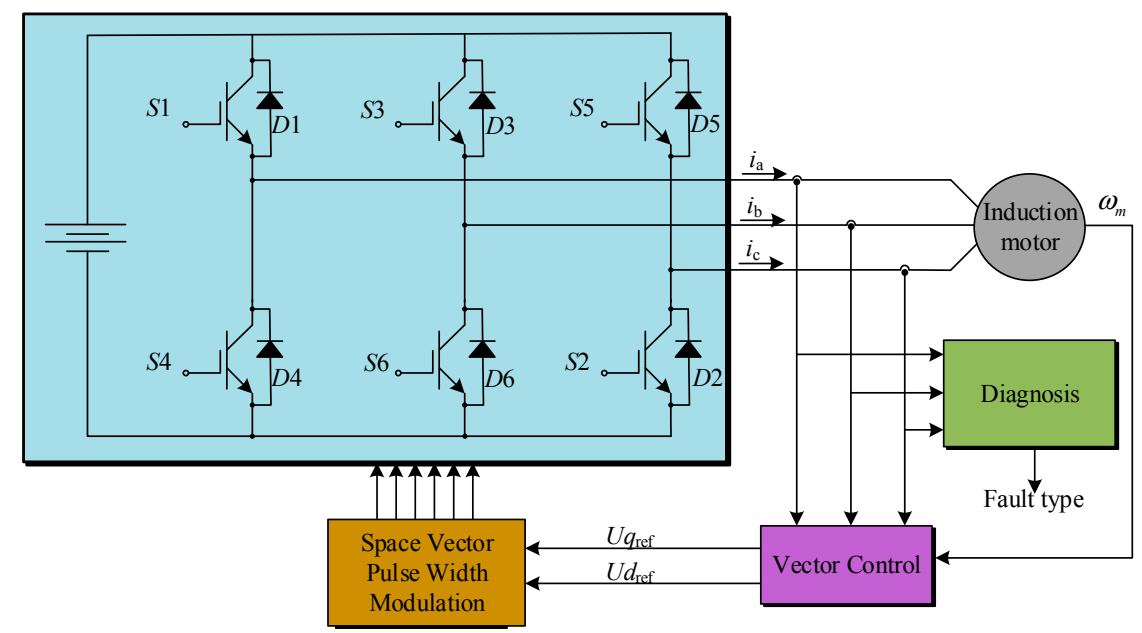

Figure 1. Schematic diagram of vector controlled traction system.

The diagnosis plan of open switch faults is shown in Figure 2. The fault detection part mainly contains two methods: SK based on CWD and WPESE. SK based on CWD is applied to detect the occurrence of fault firstly, then it is utilized to detect the fault phase with WPESE. Since the two methods could only detect the fault phase, the fault location part needs to calculate DC component of fault phase, which determines the specific locations of fault IGBT or IGBTs. Combined SK based on CWD, WPESE with DC component methods in the proposed approach, and the faulty IGBT or IGBTs can be diagnosed accurately.

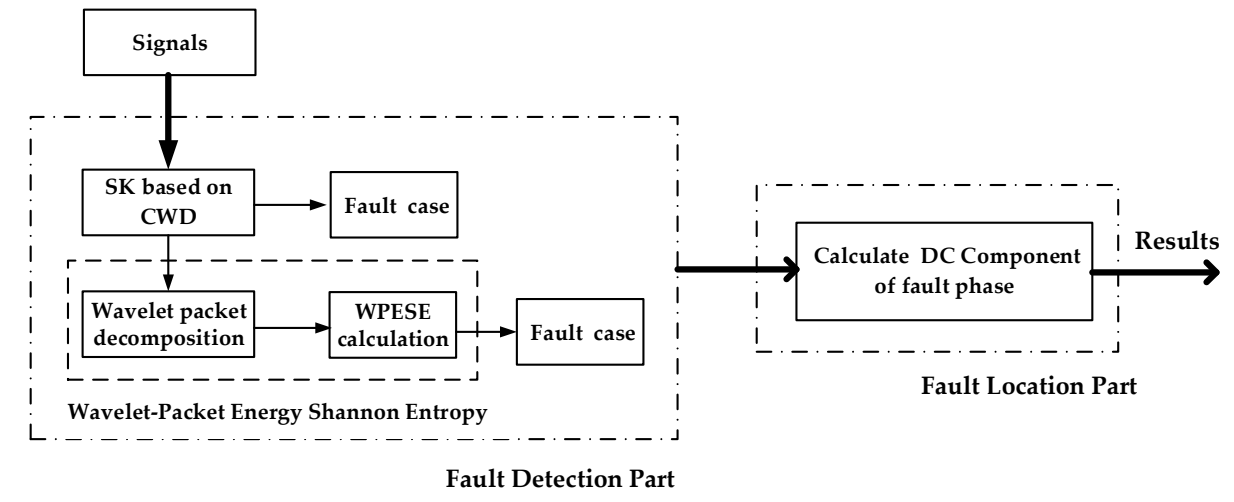

Figure 2. Diagnosis plan of open switch faults in the inverter.

\subsection{Feature Extraction}

In this paper, there are three significant features to be extracted. The diagnosis plan contains three analysis methods. Hence, these features are divided into three classes. 


\section{- SK Based on CWD Feature}

This method contains a feature named $K$, which represents the value of SK. The results of SK in fault-free, Fault $A$ and $C$ cases are shown in Figure $3 a-c$. In normal conditions, $K_{a}, K_{b}, K_{c}$ are almost equal and keep stable at about 3 in the high-frequency domain. In Fault A case with $S 1$ open or $S 4$ open, $K_{b}, K_{c}$ are similar with that in normal conditions. Meanwhile, $K_{a}$ becomes greater and the value approaches 5 . The result of Fault $C$ case with $S 1, S 4$ open shows that $K_{a}$ gets much greater than $K_{b}, K_{c}$, which approaches about 12 . Besides, Fault $B$ and $D$ cases belong to the fault condition of two IGBTs in different bridge arms. The result of Fault B case with $S 1, S 6$ open in Figure 3d shows that the increase of $K_{b}$ is small and $K_{a}$ gets greater. It is found that if a fault occurs in one phase, SK results of the phase would become much greater than under the normal condition. Thus, it is appropriate for the detection of Fault A and C cases, but it is not suitable for Fault B and D cases. It might be that if the faults occur in two IGBTs located in the different phases, the fault-free phase would be influenced simultaneously. Thus, it might cause a false alarm and affect the diagnosis results.

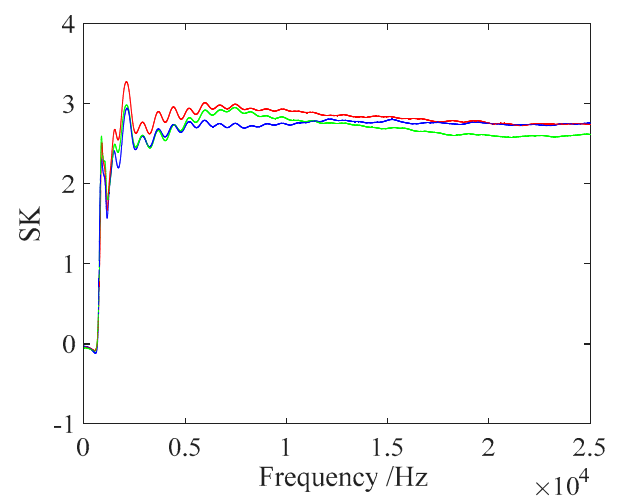

(a)

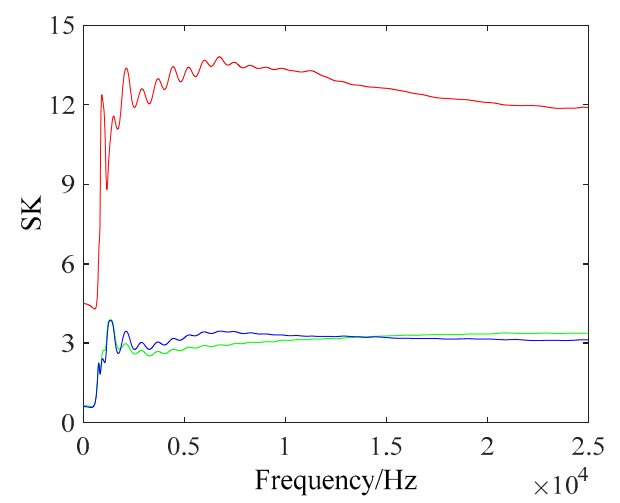

(c)

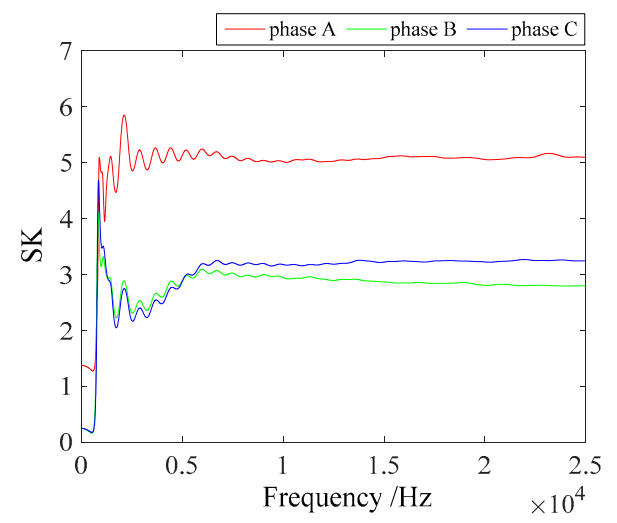

(b)

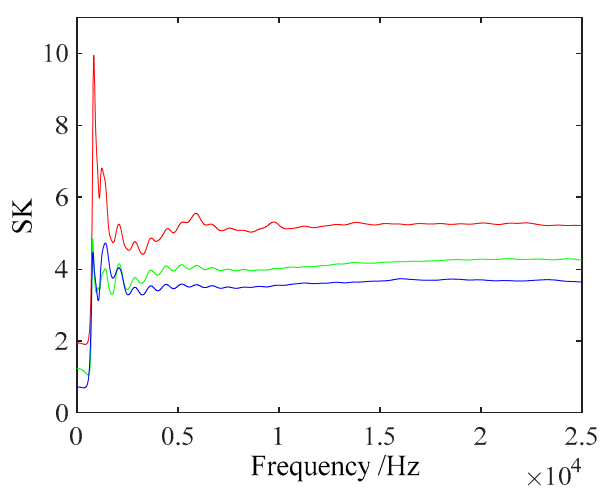

(d)

Figure 3. (a) Spectra kurtosis (SK) results in fault-free case (sampling frequency is $50 \mathrm{kHz}$ ); (b) SK results with $S 1$ open (sampling frequency is $50 \mathrm{kHz}$ ); (c) SK results with $S 1$, S4 open (sampling frequency is $50 \mathrm{kHz}$ ); (d) SK results with $S 1$, $S 6$ open (sampling frequency is $50 \mathrm{kHz}$ ).

In order to reduce the interruptions of sudden increase or decrease for SK curves, the mean value of SK is selected as the characteristic, as shown in Table 1. It is found that in fault-free case, the average values are not more than 3. If a failure of Fault A case occurs, the value of SK in fault phase increases and the average value is about 5 while the values of normal phases basically remain the same. Meanwhile, if a failure of Fault $C$ case occurs, the value of fault phase is more than 10 . Therefore, the thresholds are set to be 5 and 10 for Fault $A$ and $C$ cases, respectively. 
Table 1. The mean value of SK in different conditions.

\begin{tabular}{cccc}
\hline Conditions & Phase A & Phase B & Phase C \\
\hline Fault-free & 2.7281 & 2.6036 & 2.6282 \\
Fault A case with S1 open & 5.1584 & 2.7789 & 3.0225 \\
Fault B case with S1, S6 open & 4.5847 & 4.4133 & 3.6025 \\
Fault C case with S1, S4 open & 12.3414 & 3.0736 & 3.1208 \\
Fault D case with S1, S3 open & 6.3627 & 6.3301 & 4.7299 \\
\hline
\end{tabular}

\section{- WPESE Feature}

In the above discussion, SK cannot realize the diagnosis of Fault B and D cases, WPESE is proposed to solve the problem. There is a feature named $S_{\text {WPESE, }}$ which represents the amplitude of WPESE. Considering that the fault can be detected accurately with less time, the sliding window $L$, sliding factor $a$ and decomposition level are set to be 50 sampling points, 1 sampling point and 3 . The conditions about normal and the failure of two IGBTs $(S 1, S 6)$ with different mother wavelets are shown in Figure 4. If the condition is healthy, the entropy amplitudes of three-phase currents are small and less than 1 . Once the faults appear in the $S 1$ and $S 6$ simultaneously, WPESE results of A and B-phases become larger and much more than C-phase. It draws a conclusion that once the values of $S_{\text {WPESE }}$ in two phases get much larger, the failure would occur in them. Therefore, WPESE method can detect the fault time and fault phase by setting threshold properly. In addition, it is found that the results of three mother wavelets ("db2", "db3", "db4") are similar and they could realize the detection of open switch faults with high accuracy. In order to ensure the correctness and reduce the computational time, "db2" is selected as the mother wavelet in this paper. The simulations also indicate that, when the threshold is set to be 1, WPESE performs very well.

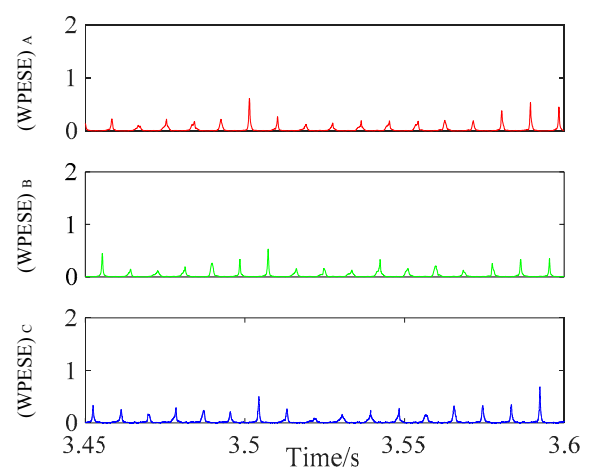

(a)

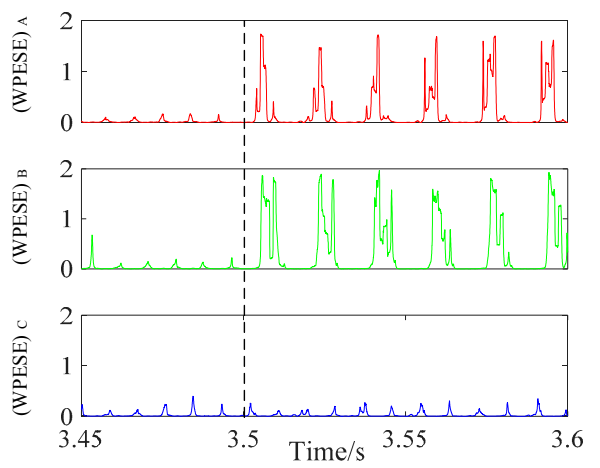

(c)

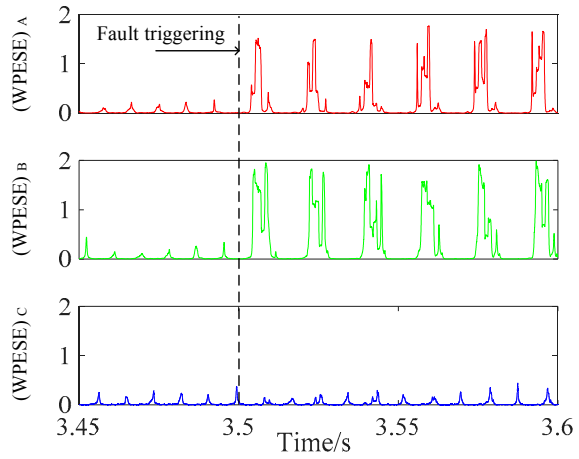

(b)

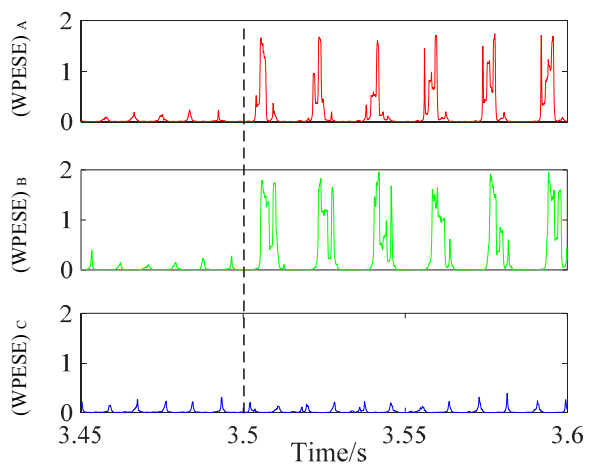

(d)

Figure 4. (a) Wavelet-packet energy Shannon entropy (WPESE) results in fault-free case; (b) WPESE results with $S 1$, S6 open with "db2"; (c) WPESE results with S1, S6 open with "db3"; (d) WPESE results with $S 1$, S6 open with "db4". 
- DC Component method

This method also contains a feature named $I_{d c}$, which means the value of DC component. DC component $I_{d c}$ can be calculated with the following formula:

$$
I_{d c}=\frac{1}{M} \sum_{m=1}^{M} I_{i}(m) i=A, B, C
$$

where, $M$ is the number of sampling points in one cycle, and $i$ represents the phase index.

Current flows and current waves of USF and LSF in A-phase are shown in Figure 5 [28]. Obviously, $I_{d c}$ is zero in fault-free case. Supposing there is a USF, the current wave can be seen in Figure $5 \mathrm{~b}, \mathrm{c}$. It is apparent that the current cannot go through $S 1$, which means that the positive current of A-phase must be zero. Meanwhile, the negative current can go through $S 4$ as before. Thus, the sampling points' value of the current will be non-positive once the fault appears. In the same way, if there is a LSF, the current waves are shown in Figure $5 \mathrm{e}, \mathrm{f}$, and the sampling points' value of the current will be non-negative after fault is triggered. Therefore, the locations of fault IGBT can be diagnosed by the calculation of DC components during the next cycle.

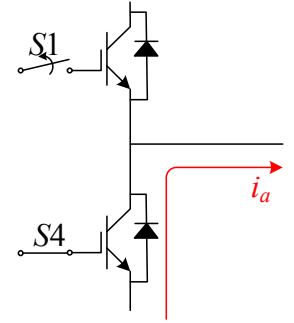

(a)

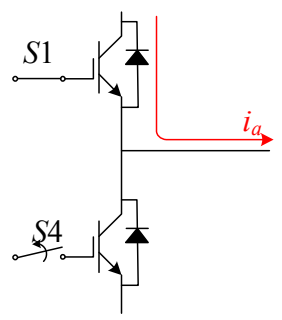

(d)

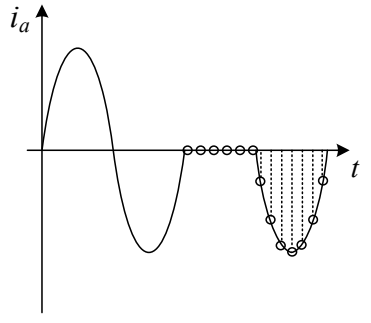

(b)

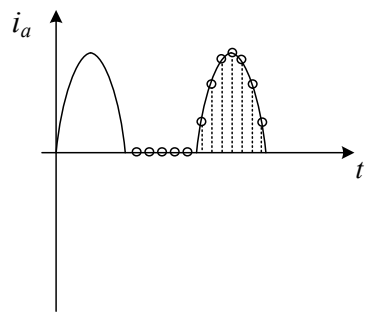

(e)

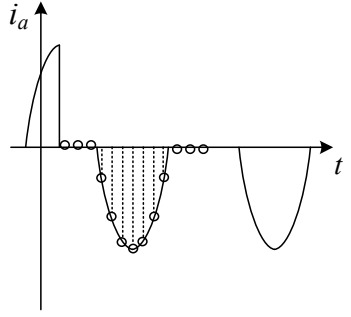

(c)

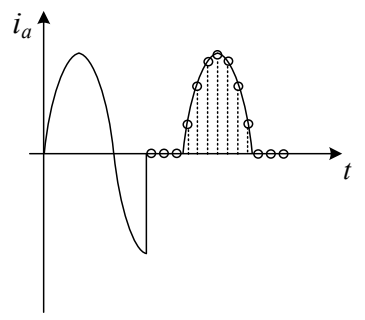

(f)

Figure 5. (a) Current flow of upper switch fault (USF); Current wave when USF occurs in(b) negative current flow; (c) Positive current flow; (d) Current flow of lower switch fault (LSF); Current wave when LSF occurs in (e) positive current flow; (f) Negative current flow.

\subsection{Diagnosis Flow}

The diagnosis flow of the proposed approach is shown in Figure 6. The three-phase currents show different characteristics in fault conditions, and a novel approach combining SK based on CWD and WPESE with the DC component method is adopted to realize the diagnosis. Firstly, SK based on CWD method is utilized to acquire the average value of $K$. It is discussed that mean $(K)$ in normal condition is not more than 3, thus, the fault would be detected if the results do not satisfy Rule 1.

If a fault is detected, Fault A and C cases can be diagnosed based on comparison of the value of SK with the set threshold. If the results of SK do not meet Rules 2 and 3, the condition belongs to a failure of two IGBTs located in different bridge arms, and WPESE is adopted. Once the results satisfy Rule 4, the condition is judged to be Fault B case. If not, it belongs to Fault D case. In Fault D case, DC components of three-phase currents are calculated. Once the polarity of two DC components is the same, IGBTs in the two bridge arms have faults. In the location part, for Fault $C$ case, it is easily to 
confirm two fault IGBTs located in fault phase. For Fault A, B and D cases, DC components of fault phases are calculated to confirm the USF or LSF. If the value of DC component is positive, it is the LSF; If is negative, it is the USF. The rules in the diagnosis flow are as follows:

Rule 1: $\operatorname{ceil}\left[\right.$ mean $\left.\left(K_{a}\right)\right]=\operatorname{ceil}\left[\right.$ mean $\left.\left(K_{b}\right)\right]=\operatorname{ceil}\left[\right.$ mean $\left.\left(K_{c}\right)\right]=3$; The $\operatorname{ceil}(x)$ function represents to take the smallest integer which is more than $x$.

Rule 2: Only one of round $\left[\operatorname{mean}\left(K_{a}\right)\right]$, round $\left[\operatorname{mean}\left(K_{b}\right)\right]$, $\operatorname{round}\left[\operatorname{mean}\left(K_{c}\right)\right]$ are more than 10 . The $\operatorname{round}(x)$ function represents to be rounded to the nearest integer.

Rule 3: Only one of round $\left[\right.$ mean $\left.\left(K_{a}\right)\right]$, round $\left[\right.$ mean $\left.\left(K_{b}\right)\right]$, round $\left[\operatorname{mean}\left(K_{c}\right)\right]$ is more than 5 , and two of $\operatorname{round}\left[\operatorname{mean}\left(K_{a}\right)\right]$, round $\left[\right.$ mean $\left.\left(K_{b}\right)\right]$, round $\left[\operatorname{mean}\left(K_{c}\right)\right]$ are 3 .

Rule 4: Only two $S_{\text {WPESE }}$ of three-phase currents are more than 1.

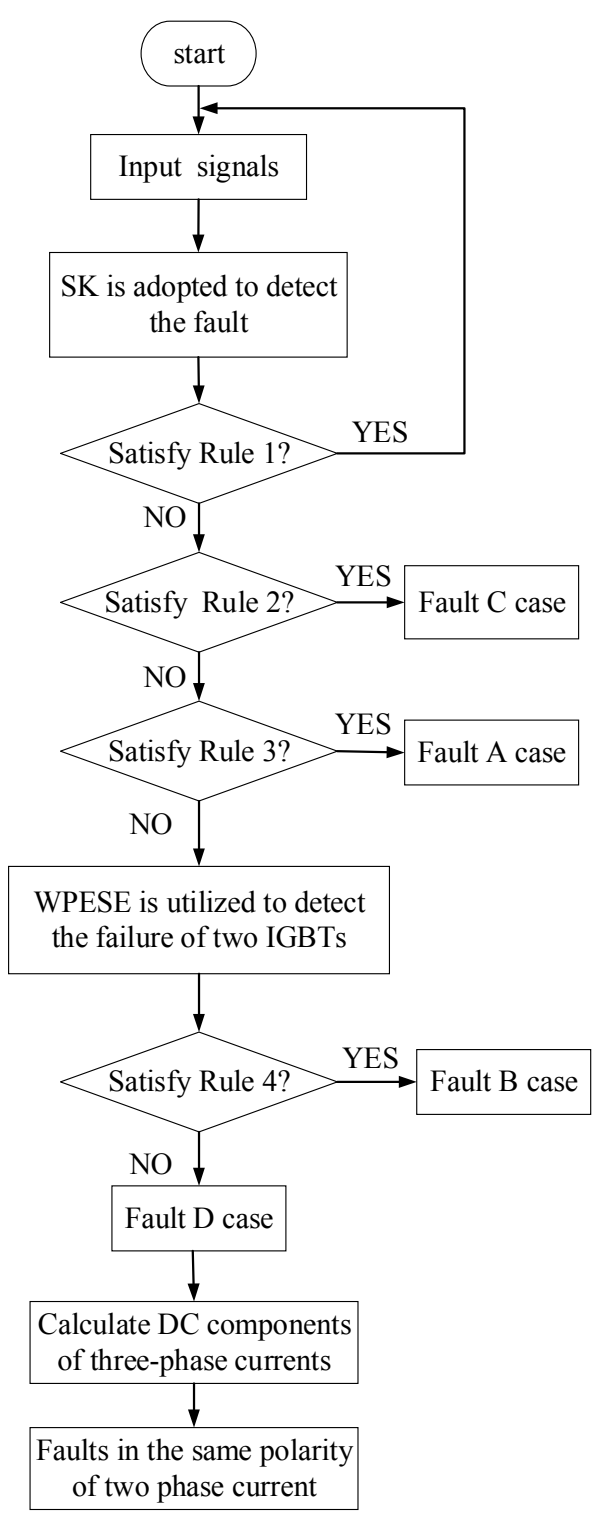

(a)

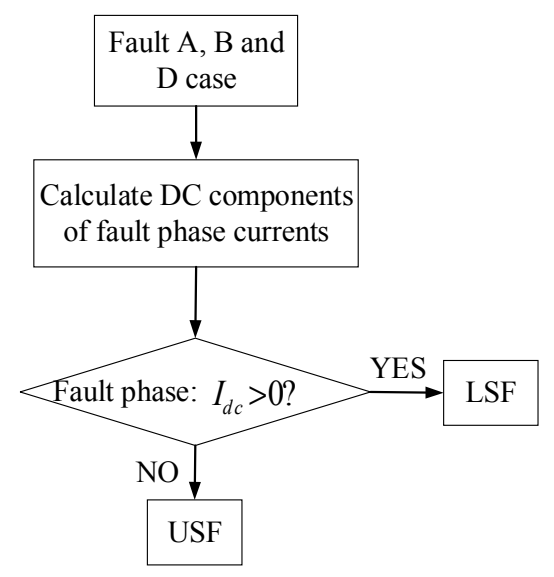

(b)

Figure 6. Fault cases diagnosis flow. (a) Fault detection part; (b) Fault location part. 


\section{Algorithm Analysis, Experiment and Discussion}

\subsection{Accuracy Analyses of Methods}

- SK Based on CWD and Window Function

SK is a time-frequency analysis algorithm. Once the window function is determined, the time-frequency resolution is fixed [22]. Thus, SK based on CWD is related with the window function. There are some typical window functions, such as rectangular, Hanning, Hamming, Blackman and Kaiser windows, etc. SK curves with Hanning and rectangular windows are shown in Figure 7a,b. It is found that the result of SK based on CWD with rectangular window has many oscillations and fluctuations. The result of SK based on CWD with Hanning window is steadier in the high-frequency domain. If the fluctuation values are too large, they might reach the set threshold and influence the diagnosis results. Hence, it is necessary to compare the smoothness of the curves.

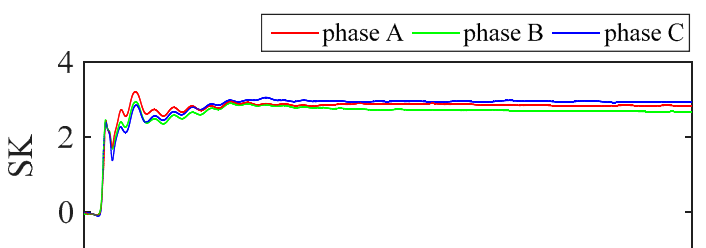

(a)

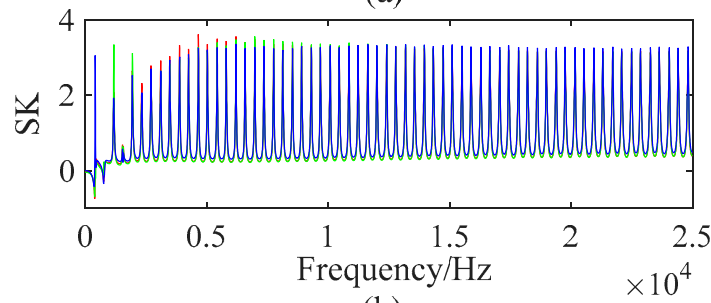

(b)

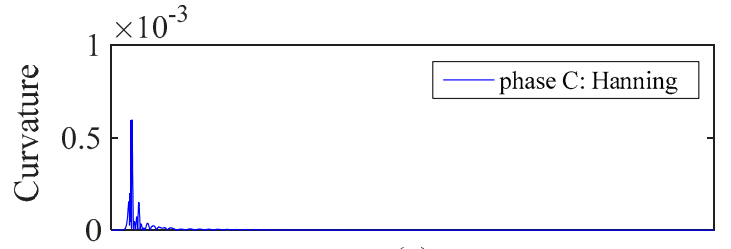

(c)

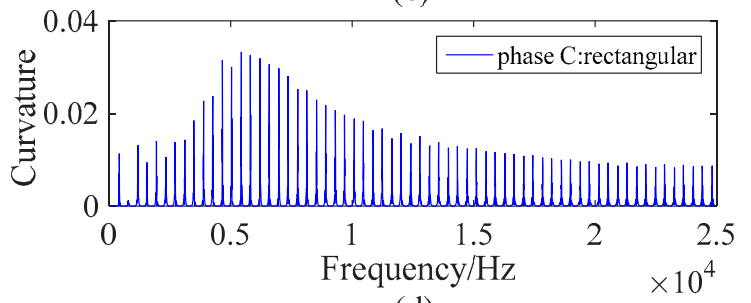

(d)

Figure 7. (a) SK result with Hanning window (sampling frequency is $50 \mathrm{kHz}$ ); (b) SK result with rectangular window (sampling frequency is $50 \mathrm{kHz}$ ); (c) The result of curvature with Hanning window; (d) The result of curvature with rectangular window.

In order to compare the smoothness of SK based on CWD curves with different window functions intuitively, the curvature is adopted. Supposing that there is a curve $y=f(x)$, the curvature $k$ can be obtained from the mathematical computation as follows:

$$
k=\frac{\left|f^{\prime \prime}(x)\right|}{\left(1+f^{\prime 2}(x)\right)^{3 / 2}}
$$

where, $f^{\prime}(x)$ is the first derivation of $x$, and $f^{\prime \prime}(x)$ is the second derivation of $x$.

The deviation of curvature is calculated and adopted to evaluate the complexity of curve shape. The smoothness of curve can be defined below [31]:

$$
\text { Smoothness }=\sum_{n=1}^{m} a b s\left(k_{n}-k_{\text {mean }}\right)
$$

where, $n$ is the index of sampling point, $m$ represents the length of data, $k_{n}$ and $k_{\text {mean }}$ are the estimated curvature of the $n$-th discrete point and the average of the estimated curvature respectively.

Based on the formula, if the value is large, the smoothness effect of curve is bad. If not, the smoothness effect of curve is good. The curvatures of C-phase current with Hanning and rectangular window are shown in Figure 7c. It is found that the change of curvature result with rectangular window is much than that with Hanning window, which is consistent with the results in Figure 7a,b. 
In addition, the average smoothness results of three-phase currents with five windows are calculated and shown in Table 2. The result with rectangular window is much larger than other windows, and results of four windows are quite similar. Actually, SK based on CWD with rectangular window is equivalent to SK without window, and the smoothing effect is not good. Hence, it is necessary to choose the efficient window function. And the results with above four windows are similar, which indicate that SK based on CWD is not sensitive with window functions.

Table 2. Average smoothness of SK with different window functions.

\begin{tabular}{cc}
\hline Window Functions & Smoothness \\
\hline Rectangular & 5.9431 \\
Hanning & 0.0347 \\
Hamming & 0.0528 \\
Blackman & 0.0464 \\
Kaiser & 0.0638 \\
\hline
\end{tabular}

\section{- SK Based on CWD and Noise}

In an actual system, signals would easily be affected by the noise, which would cause false alarms. SK is a high order cumulant with an anti-noise ability [29]. In order to reflect the performance of detection and diagnosis under fault conditions with different signal-to-noise ratio (SNR), the Gaussian white noise with SNR of 20, 30 and $40 \mathrm{~dB}$ is added to the original signals. In the discussion in Section 3.2, SK based on CWD is appropriate for diagnosis of Fault A and B cases. Hence, the normal case, Fault A and B cases are detected and diagnosed by SK based on CWD, and the results are shown in Figure 8. It is found that the results of SK based on CWD are nearly same in the normal case with different white noise, which indicate that SK could solve the false alarm problem in the fault detection. In addition, diagnosis results of Fault A and B cases could not be influenced by the noise in the system. As a consequence, the comparisons of results fully verify that SK based on CWD method has a good anti-noise performance and not easily interfered by the system noise.

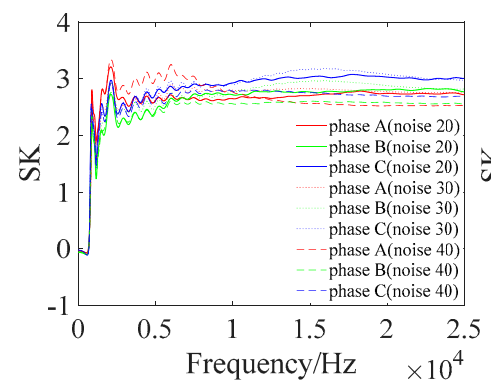

(a)

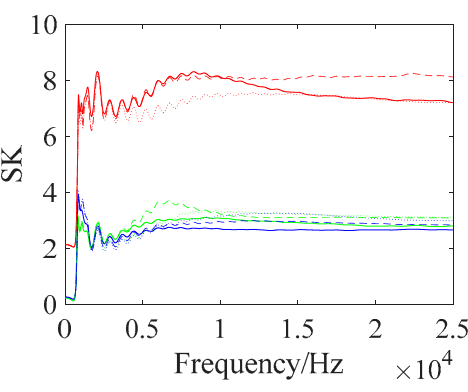

(b)

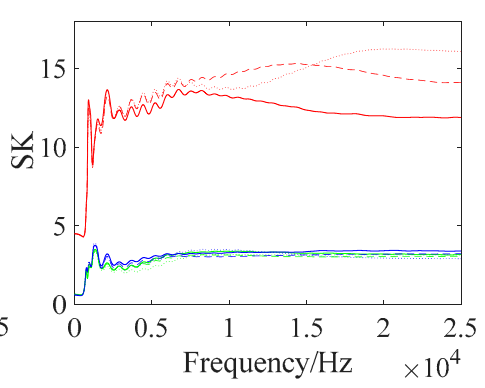

(c)

Figure 8. (a) SK results with different noise in normal case (sampling frequency is $50 \mathrm{kHz}$ ); (b) SK results with different noise in Fault A case (sampling frequency is $50 \mathrm{kHz}$ ); (c) SK results with different noise in Fault B case (sampling frequency is $50 \mathrm{kHz}$ ).

\section{- Comparison of WPESE with Empirical Mode Decomposition energy Shannon Entropy}

There are many traditional forms of entropy, such Shannon entropy, wavelet entropy, time entropy, energy entropy, etc. Recently, a new algorithm combined empirical mode decomposition (EMD) with energy Shannon entropy was proposed to detect the open switch fault of IGBT [28].

EMD method is an effective way to acquire the inherent characteristics of signals, which can separate the different scale signals to generate several series. Each series is called intrinsic mode function (IMF), and the IMFs must satisfy the following constraints [28]: 
(1) In the whole data set, the number of extrema and the number of zero-crossings must either be equal or differ at most by one;

(2) At any point, the mean value of the envelope defined by local maxima and the envelope defined by the local minima is zero.

Essentially speaking, IMFs are the extractions of signal decomposition which is achieved by the screening process. Hence, EMD method can be implemented by the following steps [32]:

(1) For a given signal $x(t)$, it is first to determine its local maxima and minima. Then cubic spline line is adopted to connect all the local maxima and minima, respectively. Thus, the upper envelope and lower envelop can be obtained and the average value is calculated as $m_{1}(t)$. Denote:

$$
h_{1}(t)=x(t)-m_{1}(t)
$$

(2) If $h_{1}(t)$ satisfies the constraints of IMF, it can be the first IMF of the original signal. Else let $x(t)=h_{1}(t)$, and repeat the Steps (1) until $h_{1 k}(t)$ satisfies the IMF constraints $\left(h_{1(k-1)}(t)-m_{1 k}(t)=h_{1 k}(t), k\right.$ is the iteration number). Denote $c_{1}(t)=h_{1 k}(t), c_{1}(t)$ is the first IMF of the original signal.

(3) Separate $c_{1}(t)$ from $x(t)$, and the residual can be obtained as below:

$$
r_{1}(t)=x(t)-c_{1}(t)
$$

Let $r_{1}(t)$ be the original signal instead of $x(t)$, and repeat the Steps (1) and (2), the second IMF $c_{2}(t)$ can be acquired. Based on that, repeat the steps above until the residual is monotonous. The original signal can be expressed as:

$$
x(t)=\sum_{i=1}^{n} c_{i}(t)+r_{n}(t)
$$

where, $r_{n}$ represents the average trend of the signal, and IMF components $c_{i}, i=1,2, \ldots, n$ represent the different frequency components from high to low.

The probability and empirical mode decomposition Shannon entropy (EMDESE) can be obtained as Equations (15) and (16):

$$
\begin{gathered}
p_{i}=c_{i} / \sum_{j=1}^{n} c_{j} \\
\text { EMDESE }=-\sum_{i=1}^{n} p_{i} \log p_{i}
\end{gathered}
$$

The results of EMD and EMDESE are shown in Figure 9. The sliding window $L=150$ sampling points, sliding factor $a=1$ sampling points and decomposed layer $y=7$. It is found that EMDESE result of A-phase is more than the threshold before fault trigging, which would cause false alarms and affect the accuracy of the diagnosis. Thus, compared with EMDESE, WPESE is more appropriate for the diagnosis of faults.

Based on the above discussion, SK based on CWD is not sensitive with window functions and has a strong anti-noise ability. Besides, through the comparison of WPESE with EMDESE, the diagnosis result of WPESE is more reliable. Therefore, the approach could solve the false alarm problem which could be caused by the system noise and has good robustness. 


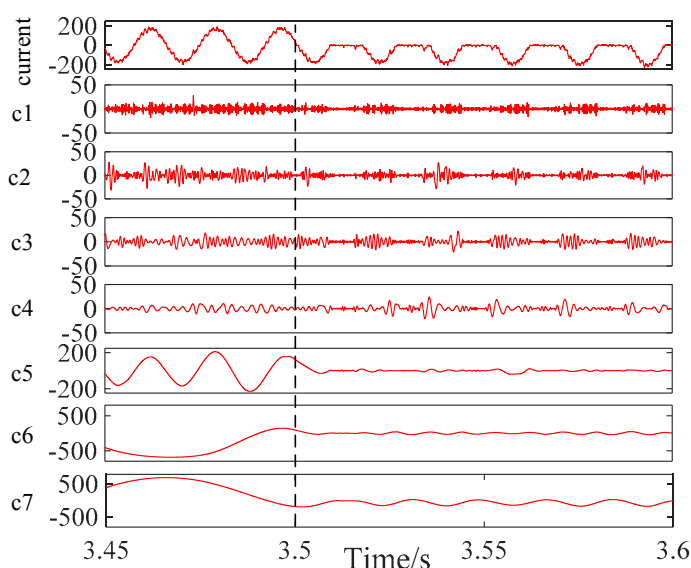

(a)

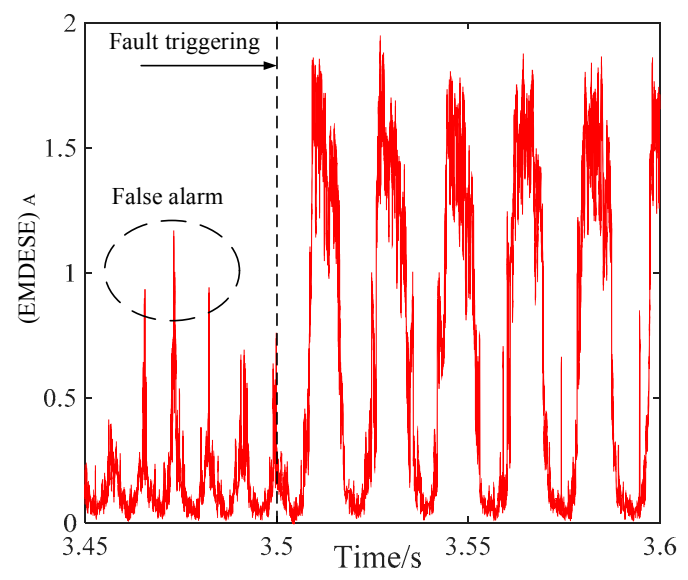

(b)

Figure 9. (a) Empirical mode decomposition (EMD) results of A-phase fault current with S1 open; (b) empirical mode decomposition Shannon entropy (EMDESE) results of A-phase fault current with S1 open.

\subsection{Experiment Results}

To further validate the performance of the proposed method, some real-time online experiments about the open switch faults of IGBTs in the inverter are implemented by the RT-LAB (v11.0.8.13) platform. Through the combination of an OP5600 real-time digital simulator (OPAL-RT technologies, Canada) and its upper computer interface, the validation can be carried out. The structure diagram and the real products of real-time online simulation system are shown in Figure 10a,b, respectively.

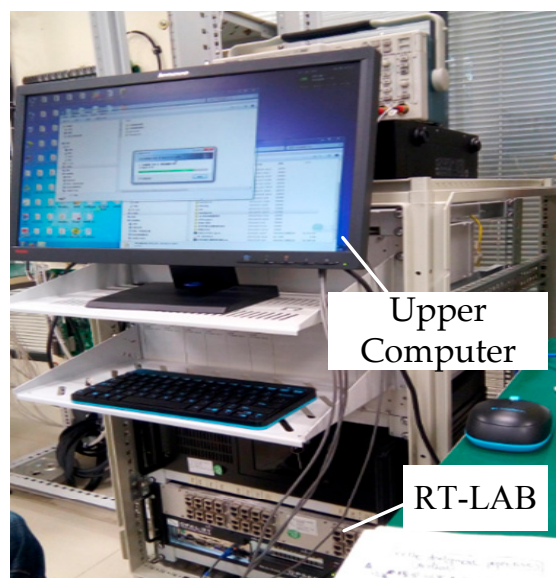

(a)

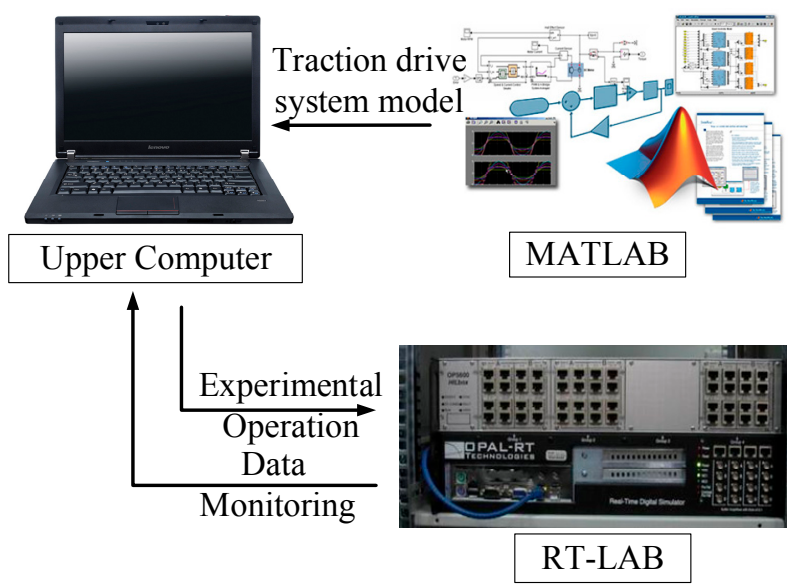

(b)

Figure 10. Real-time online simulation system. (a) Real products of the system; (b) Structure diagram of the system.

First of all, the model of closed-loop controlled traction drive system is established in SIMULINK (MATLAB 2011b). There are three main parts of this model, namely, the traction motor (a squirrel cage induction motor), the DC voltage fed inverter, and the controller. The overall sketch can be seen in Figure 1. The parameters of the traction motor are shown in Table 3. The operating switch frequency of the inverter is $500 \mathrm{~Hz}$, and the fall time and tail of time for each IGBT are $1 \mu \mathrm{s}$ and $2 \mu \mathrm{s}$, respectively. The control strategy is vector control which is a popular strategy in traction drive system, and the modulation method is space vector pulse width modulation. 
Table 3. Parameters of the traction motor.

\begin{tabular}{cccc}
\hline Parameter & Value & Parameter & Value \\
\hline Stator resistance $/ \mathrm{R}_{\mathrm{s}}$ & $0.15 \Omega$ & Inertia $/ \mathrm{J}$ & $10 \mathrm{~kg} \cdot \mathrm{m}^{2}$ \\
Stator leakage inductance $/ \mathrm{L}_{\mathrm{s}}$ & $0.00142 \mathrm{H}$ & Nominal line voltage & $2700 \mathrm{~V}$ \\
Rotator resistance $/ \mathrm{R}_{\mathrm{r}}$ & $0.16 \Omega$ & Nominal frequency & $138 \mathrm{~Hz}$ \\
Rotator leakage inductance $/ \mathrm{L}_{\mathrm{s}}$ & $0.006 \mathrm{H}$ & Pole pairs & 2 \\
Mutual inductance $/ \mathrm{L}_{\mathrm{m}}$ & $0.0254 \mathrm{H}$ & Nominal power & $\mathrm{kW}$ \\
\hline
\end{tabular}

Then, the model is transformed into the real-time OP5600 digital simulator, where the validation is performed. To create the condition where the IGBT is open, the drive pulse of the corresponding IGBT will be removed. For example, to make $S 1$ open at $3.5 \mathrm{~s}$, the pulse of $S 1$ will be removed at $3.5 \mathrm{~s}$, then $S 1$ keeps off. An upper computer is utilized as the data monitor, and the sampling frequency is $50 \mathrm{kHz}$. It keeps receiving the data from RT-LAB, which makes it possible to perform our method to diagnose the open switch faults of IGBT. Finally, aiming at the four open-switch faults and the fault-free case, in total five conditions have been considered for further discussion.

\subsubsection{Fault-Free Case}

In a normal condition, three-phase currents and SK based on CWD curves of currents are depicted in Figure 11. The amplitudes of currents are the same. The average values of SK based on CWD are all less than 3 and satisfy Rule 1, thus, the results do not meet the fault condition, which verifies that the system is in the fault-free case.

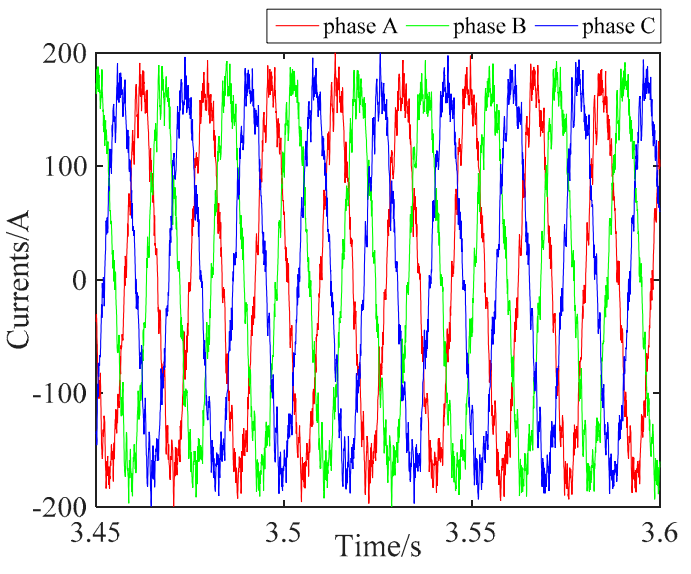

(a)

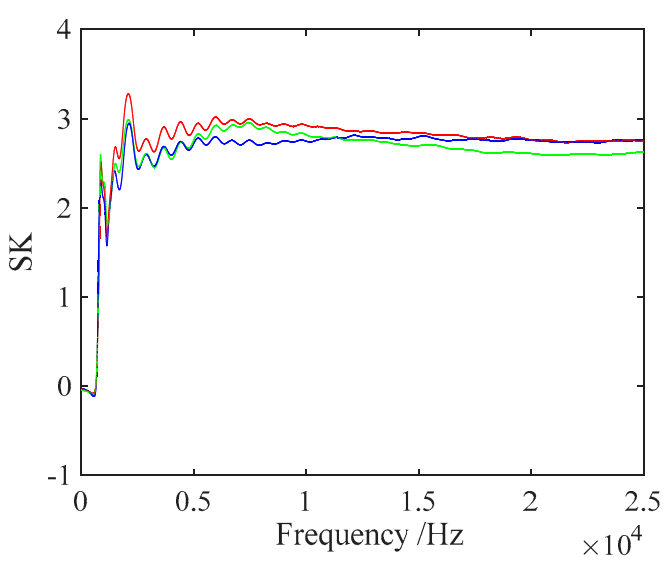

(b)

Figure 11. (a) Three-phase currents in fault-free case; (b) SK results in fault-free condition.

\subsubsection{Fault A Case}

The open switch fault of an IGBT (S1) is carried out, and three-phase fault currents can be seen in Figure 12a. It is found the positive half cycle of A-phase current becomes zero at the fault time. SK based on CWD is utilized to detect the fault firstly, and curves of results are shown in Figure 12b. The average values of SK results are calculated and satisfy Rule 3, which indicates that the fault occurs in A-phase. Then, DC component of fault phase is calculated, as shown in Figure 12c. Since the DC component is negative after fault triggering, the fault can be diagnosed in the USF of A-phase, i.e., S1. 


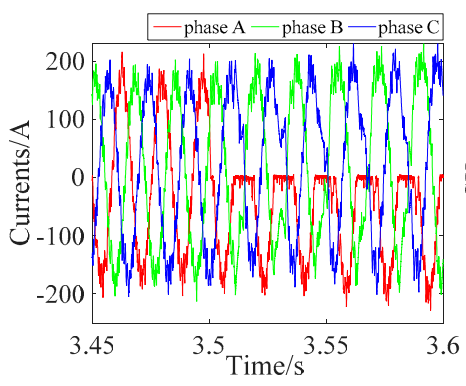

(a)

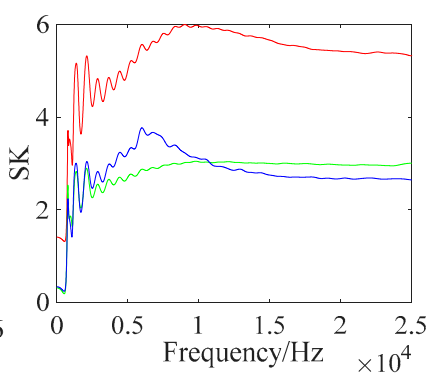

(b)

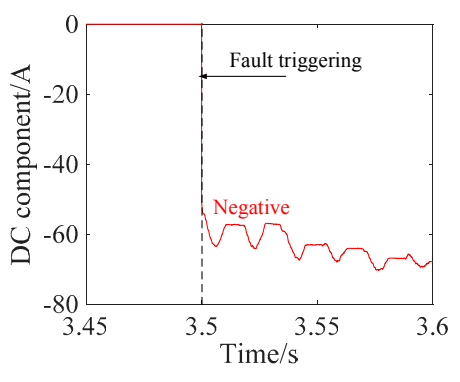

(c)

Figure 12. (a) Three-phase currents with $S 1$ open; (b) Results of SK with $S 1$ open; (c) DC component of A-phase with $S 1$ open.

\subsubsection{Fault B Case}

In this condition, open switch fault of IGBTs $(S 1, S 6)$ is carried out, and fault currents can be seen in Figure 13a. The current of A-phase is non-positive while B-phase is non-negative. Also, SK based on CWD is applied to detect the fault firstly, and result is shown in Figure 13b. It indicates that a fault occurs in IGBT, and the result does not satisfy Rules 2 and 3. Hence, the WPESE method is adopted to acquire $S_{\text {WPESE }}$ of three-phase currents and the consequences are shown in Figure 13c. According to the results, $S_{\text {WPESE }}$ of A and B-phases are both more than the threshold while $S_{\text {WPESE }}$ of C-phase is less than the threshold. Since the results satisfy Rule 4, it is judged to be the Fault B case, and the faults appear in the A and B-phases. In addition, the DC component of the A-phase is negative and that of B-phase is positive in Figure 13d. Therefore, it turns out that the fault is diagnosed in the USF of A-phase and the LSF of B-phase, i.e., S1 and S6.

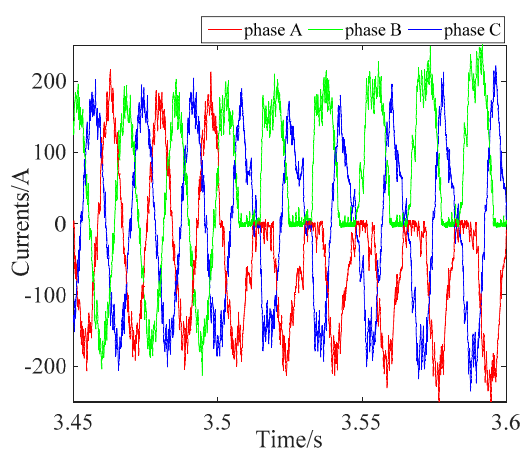

(a)
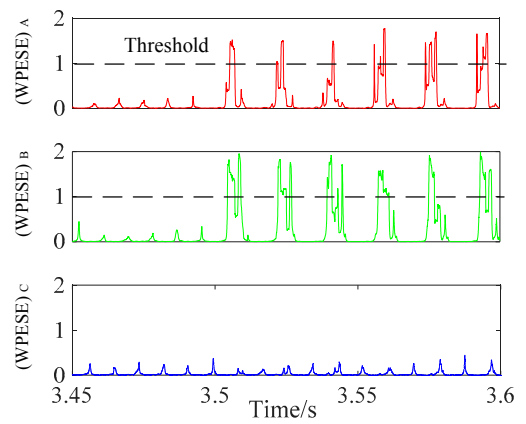

(c)

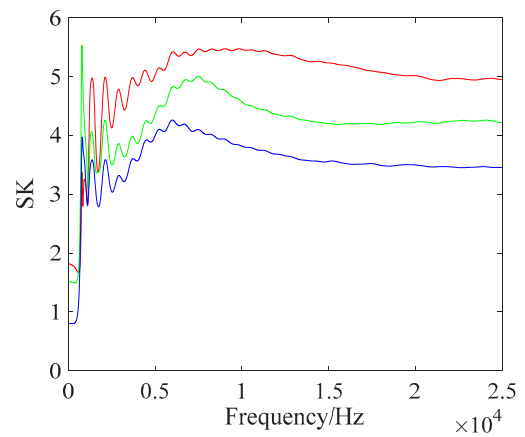

(b)

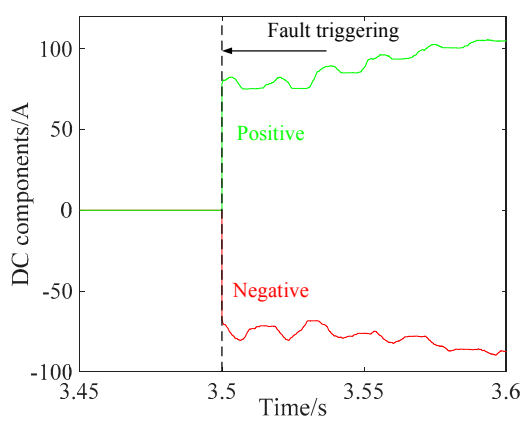

(d)

Figure 13. (a) Three-phase currents with S1, S6 open; (b) SK results with S1, S6 open; (c) DC components of three-phase currents with $S 1, S 6$ open; (d) WPESE results of three-phase currents. 


\subsubsection{Fault C Case}

Compared with Fault A and B cases, Fault $C$ case is easier to be diagnosed. The open switch fault of A-phase $(S 1, S 4)$ is carried out and fault currents are shown in Figure 14a. Obviously, the currents of A-phase become zero when the fault occurs. The results of three-phase currents are acquired by SK based on CWD in Figure 14b. Once the fault is triggered, the value of SK in A-phase is much more than B and C-phases, which approaches about 12 and meets Rule 2. Therefore, the fault can be diagnosed and judged to be Fault C case and the locations are the USF and LSF of A-phase, i.e., S1 and S4.

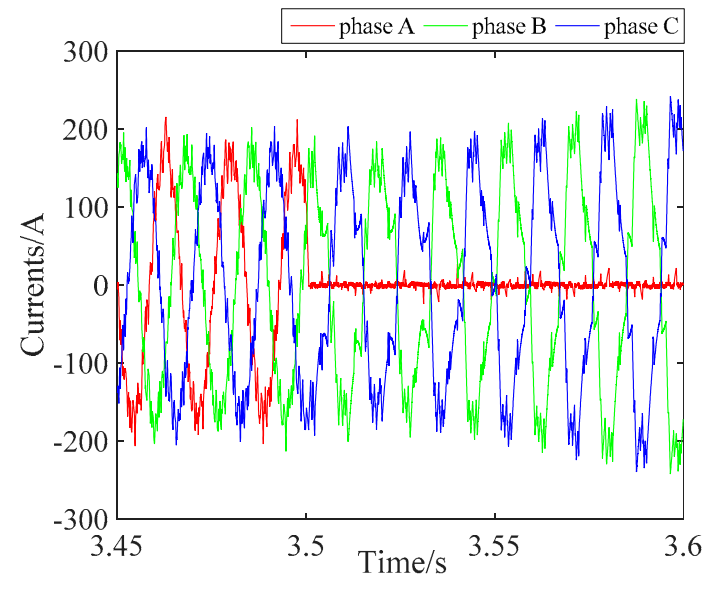

(a)

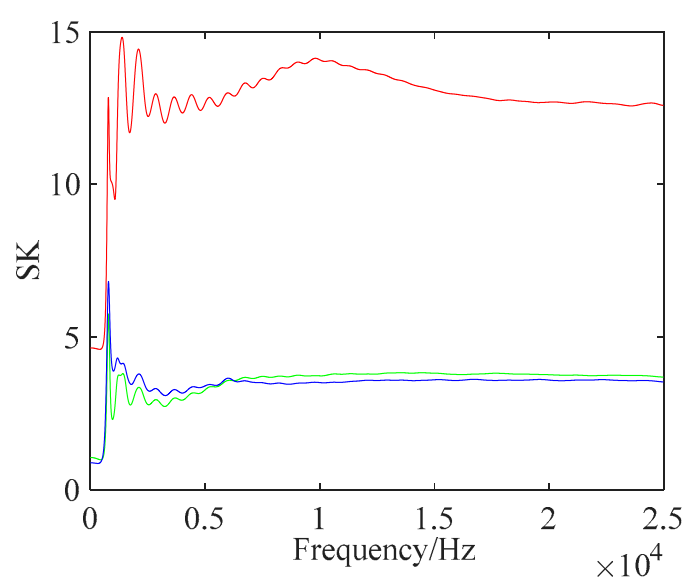

(b)

Figure 14. (a) Three-phase currents with $S 1, S 4$ open; (b) SK results with $S 1$, $S 4$ open.8.

\subsubsection{Fault D Case}

In Fault D case, a fault condition of IGBTs $(S 1, S 3)$ is carried out. The fault currents can be seen in Figure 15a. The currents of A and B-phases are non-positive and current of C-phase is non-negative. This fault case is special and it is easily to cause the false alarm. Firstly, SK based on CWD is adopted and the results cannot satisfy Rule 2 and Rule 3 in Figure 15b. Thus, the WPESE method is applied to diagnose the fault condition in Figure 15c. It is found that $S_{\text {WPESE }}$ of three-phase currents are all more than the set threshold. Based on the proposed algorithm, the condition is judged to be the Fault D case. Then, diagnosis of IGBTs is realized by calculating DC components of three-phase currents, and the results are shown in Figure 15d. The results of DC components show that the DC components of A and B-phases are negative while C-phase is positive, thus, the faults can be diagnosed in A and B-phases, and the locations are the USF of fault legs, i.e., S1, S3.

In conclusion, the experimental results verify the effectiveness of the proposed fault diagnosis method. Compared to the existing fault diagnosis methods, the proposed approach can realize the diagnosis of the faults more effectively and it requires no additional devices. 


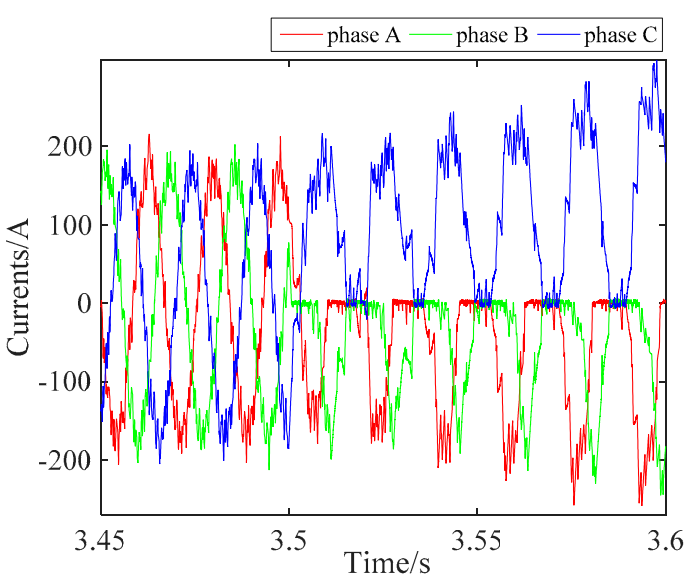

(a)
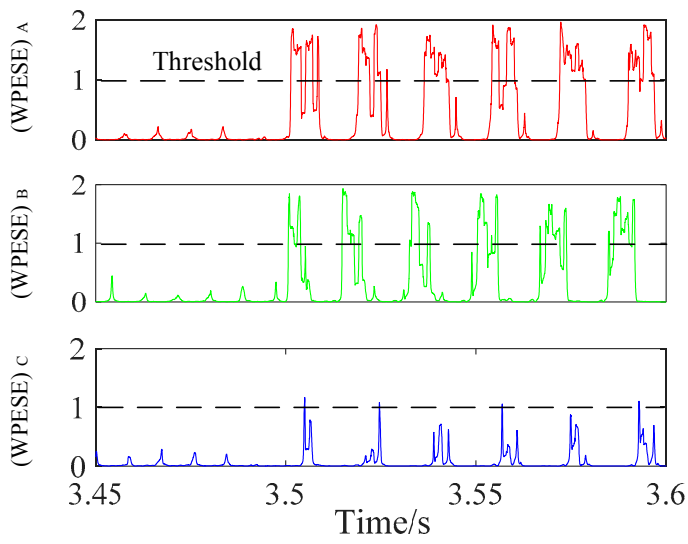

(c)

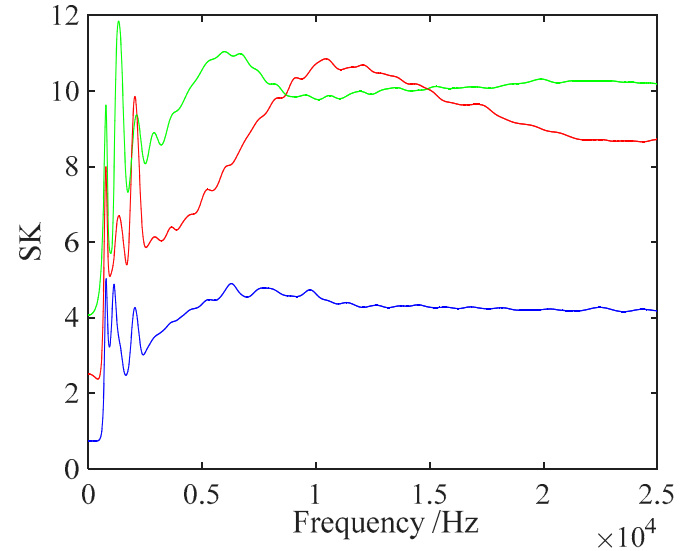

(b)

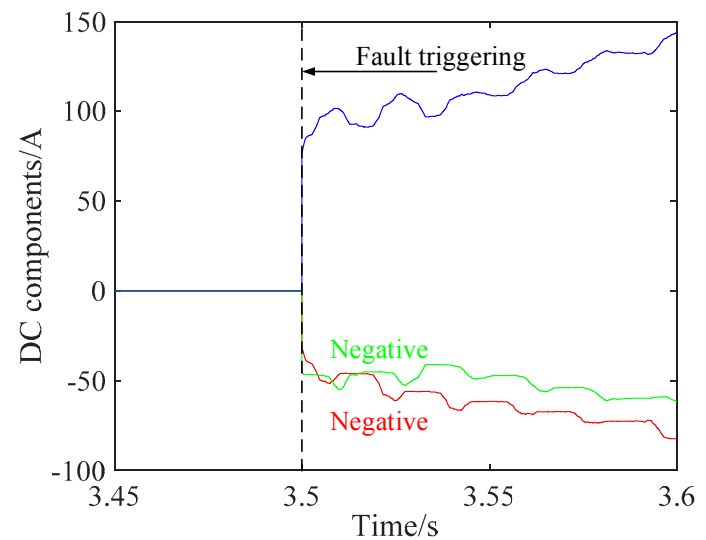

(d)

Figure 15. (a) Three-phase currents with $S 1, S 3$ open; (b) Results of SK with $S 1$, S3 open; (c) Results of WPESE in three-phase currents with $S 1, S 3$ open; (d) DC components of three-phase currents with $S 1$, S3 open.

\section{Conclusions}

In this paper, aiming at detecting open switch faults in an IGBT or IGBTs, a novel diagnosis approach is proposed based on the analyses of three-phase currents. The approach mainly contains two parts, namely the fault detection part with SK based on the CWD and WPESE methods, and the fault location part with DC component method. In the fault detection part, SK based on CWD is utilized to detect the fault firstly and combined with WPESE to detect the fault phase. In the fault location part, the DC components of the fault phase are calculated for determining the specific locations of faulty IGBT or IGBTs. In addition, the experiments on the RT-LAB platform of fault-free and four typical fault cases are implemented, and the results show that the proposed approach can diagnose the faults feasibly and effectively. The properties of the method can be summarized as follows:

(1) SK based on CWD is not sensitive to the window functions and has an anti-noise ability, WPESE could diagnose the fault with accuracy compared with EMDESE. Therefore, the approach could solve the false alarm problem which would be caused by the system noise and has good robustness.

(2) Compared with some previous methods, the method can realize the diagnosis of all single and two IGBTs fault cases and it can detect a fault and locate the open switch fault IGBTs with high accuracy.

(3) The method is easily operated and requires no additional devices. 
Acknowledgments: This study is partly supported by National Nature Science Foundation of China (No. U1434203, 51377136) and Sichuan Province Youth Science and Technology Innovation Team (No. 2016TD0012).

Author Contributions: The individual contribution of each co-author to the reported research and writing of the paper are as follows. Zhigang Liu and Shuangshuang Lin conceived the idea, Keting Hu and Shuangshuang Lin performed experiments and data analysis, and all authors wrote the paper. All authors have read and approved the final manuscript.

Conflicts of Interest: The authors declare no conflict of interest.

\section{Nomenclature}

$\begin{array}{ll}\text { CWD } & \text { Choi-Williams Distribution } \\ \text { EMD } & \text { Empirical mode decomposition } \\ \text { EMDESE } & \text { Empirical mode decomposition energy Shannon entropy } \\ \text { IGBT } & \text { Isolated Gate Bipolar Transistor } \\ \text { LSF } & \text { Lower Switch Fault } \\ \text { SNR } & \text { Signal-to-noise ratio } \\ \text { SK } & \text { Spectral kurtosis } \\ \text { SP } & \text { Signal processing } \\ \text { SPD } & \text { Semiconductor power devices } \\ \text { STFT } & \text { Short Time Fourier Transform } \\ \text { USF } & \text { Upper Switch Fault } \\ \text { WPESE } & \text { Wavelet-Packet Energy Shannon Entropy } \\ \text { WT } & \text { Wavelet Transform } \\ \text { WVD } & \text { Wigner-Ville Distribution }\end{array}$

\section{References}

1. Smet, V.; Forest, F.; Huselstein, J.J.; Richardeau, F.; Khatir, Z.; Lefebvre, S.; Berkani, M. Ageing and failure modes of IGBT modules in high-temperature power cycling. IEEE Trans. Ind. Electron. 2011, 58, 4931-4941. [CrossRef]

2. Yang, S.; Bryant, A.; Mawby, P.; Xiang, D.; Ran, L.; Tavner, P. An industry-based survey of reliability in power electronic converters. IEEE Trans. Ind. Appl. 2011, 47, 1441-1451. [CrossRef]

3. An, Q.T.; Sun, L.Z.; Zhao, K.; Sun, L. Switching function model-based fast-diagnostic method of open- switch faults in inverters without sensors. IEEE Trans. Power Electron. 2011, 26, 119-126. [CrossRef]

4. Wang, Q.; Wang, Y.R.; Zhang, Z.F.; Song, Z. A diagnosis method for inverter open-circuit faults of brushless DC motor driver systems. Proc. CSEE 2013, 33, 114-120.

5. Ribeiro, R.L.D.; Jacobina, C.B.; da Silva, E.R.C.; Lima, A.M.N. Fault detection of open-switch damage in Voltage-Fed PWM motor drive systems. IEEE Trans. Power Electron. 2013, 18, 587-593. [CrossRef]

6. Cui, B.W.; Ren, Z. Fault detection and isolation of inverter based on FFT and neural network. Trans. CES 2006, 21, 37-43.

7. Cui, J.; Wang, Q.; Gong, C.Y. Inverter power switch fault diagnosis technique research based on wavelet and Concordia transform. Proc. CSEE 2015, 35, 3110-3116.

8. Keswani, R.A.; Suryawanshi, H.M.; Ballal, M.S. Multi-resolution analysis for converter switch faults identification. IET Power Electron. 2015, 8, 783-792. [CrossRef]

9. Aktas, M.; Turkmenoglu, V. Wavelet-based switching faults detection in direct torque control induction motor drives. IET Sci. Meas. Technol. 2010, 4, 303-310. [CrossRef]

10. Kim, D.E.; Lee, D.C. Fault diagnosis of three-phase PWM inverters using wavelet and SVM. J. Power Electron. 2009, 9, 377-385.

11. Estima, J.O.; Cardoso, A.J.M. A new approach for real-time multiple open-circuit fault diagnosis in voltage-source inverters. IEEE Trans. Ind. Appl. 2011, 47, 2487-2494. [CrossRef]

12. Zhang, J.H.; Zhao, J.; Zhou, D.H.; Huang, C.G. High-performance fault diagnosis in PWM voltage-source inverters for vector-controlled induction motor drives. IEEE Trans. Power Electron. 2014, 29, 6087-6099. [CrossRef] 
13. Estima, J.O.; Freire, N.M.A.; Cardoso, A.J.M. Recent Advances in Fault Diagnosis by Park's Vector Approach. In Proceedings of the 2013 IEEE Workshop on Electrical Machines Design Control and Diagnosis, Paris, France, 11-12 March 2013; pp. 279-288.

14. Ferracuti, F.; Giantomassi, A.; Iarlori, S.; Ippoliti, G.; Longhi, S. Electric motor defects diagnosis based on kernel density estimation and Kullback-Leibler divergence in quality control scenario. Eng. Appl. Artif. Intell. 2015, 44, 25-32. [CrossRef]

15. Villecco, F.; Pellegrino, A. Entropic measure of epistemic uncertainties in multibody system models by axiomatic design. Entropy 2017, 19, 291. [CrossRef]

16. Villecco, F.; Pellegrino, A. Evaluation of uncertainties in the design process of complex mechanical systems. Entropy 2017, 19, 475. [CrossRef]

17. Wang, D.; Tsui, K.L. Dynamic Bayesian wavelet transform: New methodology for extraction of repetitive transients. Mech. Syst. Signal Process. 2017, 88, 137-144. [CrossRef]

18. Wang, D.; Peter, W.T.; Tsui, K.L. An enhanced Kurtogram method for fault diagnosis of rolling element bearings. Mech. Syst Signal Process. 2013, 35, 176-199. [CrossRef]

19. Jia, F.; Lei, Y.; Shan, H.; Lin, J. Early fault diagnosis of bearings using an improved spectral kurtosis by maximum correlated kurtosis deconvolution. Sensors 2015, 15, 29363-29377. [CrossRef] [PubMed]

20. Dwyer, R. Detection of non-Gaussian signals by frequency domain kurtosis estimation. In Proceedings of the IEEE International Conference on Acoustics, Speech, and Signal Processing (ICASSP 1983), Boston, MA, USA, 14-16 April 1983; pp. 607-610.

21. Antoni, J. The spectral kurtosis: A useful tool for characterising non-stationary signals. Mech. Syst. Signal Process. 2006, 20, 282-307. [CrossRef]

22. Liu, Z.G.; Zhang, Q.G. An approach to recognize the transient disturbances with spectral kurtosis. IEEE Trans. Instrum. Meas. 2014, 63, 46-55. [CrossRef]

23. Wang, D. An extension of the infograms to novel Bayesian inference for bearing fault feature identification. Mech. Syst. Signal Process. 2016, 80, 19-30. [CrossRef]

24. Gao, Y.; Villecco, F.; Li, M.; Song, W. Multi-Scale Permutation Entropy Based on Improved LMD and HMM for Rolling Bearing Diagnosis. Entropy 2017, 19, 176. [CrossRef]

25. Guo, Y.; Xu, Q.; Li, P.; Sbert, M.; Yang, Y. Trajectory Shape Analysis and Anomaly Detection Utilizing Information Theory Tools. Entropy 2017, 19, 323. [CrossRef]

26. Liu, Z.G.; Cui, Y.; Li, W.H. Combined power quality disturbances recognition using wavelet packet entropies and S-transform. Entropy 2015, 17, 5811-5828. [CrossRef]

27. Liu, Z.G.; Han, Z.W.; Zhang, Y. Multiwavelet packet entropy and its application in transmission line fault recognition and classification. IEEE Trans. Neural Netw. Learn. Syst. 2014, 25, 2043-2052. [CrossRef] [PubMed]

28. Hu, K.T.; Liu, Z.G.; Lin, S.S. Wavelet entropy-based traction inverter open switch fault diagnosis in high-speed railways. Entropy 2016, 18, 78. [CrossRef]

29. Zhu, L.; Liu, Z.; Hu, Q.; Zhang, Q. School of Electrical Engineering, Southwest Jiaotong University. Recognition of transient power quality disturbances based on CWD spectral kurtosis. Electr. Power Autom. Equip. 2014, 34, 125-131.

30. Campos-Delgado, D.U.; Espinoza-Trejo, D.R. An observer-based diagnosis scheme for single and simultaneous open-switch faults in induction motor drives. IEEE Trans. Ind. Electron. 2011, 58, 671-679. [CrossRef]

31. Liu, Z.G.; Cui, Y.; Li, W.H. A classification method for complex power quality disturbances using EEMD and rank wavelet SVM. IEEE Trans. Smart Grid 2015, 6, 1678-1685. [CrossRef]

32. Ding, W.L.; Gao, X.Y.; Su, Y.P.; Li, X.L. A movement smoothness measuring algorithm based on curvature estimation. High Technol. Lett. 2015, 25, 835-842.

(C) 2017 by the authors. Licensee MDPI, Basel, Switzerland. This article is an open access article distributed under the terms and conditions of the Creative Commons Attribution (CC BY) license (http:/ / creativecommons.org/licenses/by/4.0/). 